\title{
FACTORES SOCIOECONÓMICOS QUE INTERVIENEN EN EL DESEMPEÑO ACADÉMICO DE LOS ESTUDIANTES UNIVERSITARIOS DE LA FACULTAD DE CIENCIAS HUMANAS DE LA UNIVERSIDAD AUTÓNOMA DE BAJA CALIFORNIA
}

\author{
Nereyda G. Armenta, Claudia C. Pacheco y Erika D. Pineda \\ Universidad Autónoma de Baja California, México \\ (RECIBIDO 21/ 11/ 2007, ACEPTADO 14/05/2008)
}

\begin{abstract}
RESUMEN
La presente investigación pretende conocer de qué manera afectan los factores socioeconómicos en el desempeño académico de los estudiantes universitarios de la Facultad de Ciencias Humanas de la Universidad Autónoma de Baja California (UABC), con el objetivo de determinar cuál es la causa del bajo rendimiento académico. Se trabajó con una muestra de 60 estudiantes, a los cuales se les aplicó una encuesta que consta de 21 preguntas de opción múltiple. Los resultados obtenidos muestran que las hipótesis planteadas son nulas ya que ninguno de los factores afecta el desempeño académico de los universitarios.
\end{abstract}

Palabras clave: Contexto, Influencia y Causas.

\begin{abstract}
In the present investigation one tries to know about what way they affect the socioeconomic factors in the academic performance of the university students of the facultyof human sciences of the UABC, with the aim to determine which is the reason of the low academic performance. I work with a sample of 60 students to which apply a survey that consists of 21 questions of multiple option. The obtained results show that the raised hypotheses are void since none of the factors affects the academic performanc of the university students.
\end{abstract}

Keywords: Context, Influence and Causes.

\section{INTRODUCCIÓN}

En el siguiente documento se presenta una investigación realizada con alumnos que pertenecen a la Facultad de Ciencias Humanas de la Universidad Autónoma de Baja California. Se implementó varias hipótesis de lo que la sociedad cree que influye en el desempeño de los estudiantes y basándose en éstas se aplicó el instrumento de medición y así se obtuvieron los resultados que se presentan. En este trabajo de investigación, se adoptan diferentes posturas de autores que hablan de algunos de los factores que afectan 
el desempeño académico de estudiantes y se utilizó el método cuantitativo; para el análisis de los datos se utilizó la base de datos SPSS.

\section{Justificación}

El tema: "Factores socioeconómicos que intervienen en el desempeño académico de los estudiantes universitarios" es interesante ya que los estudiantes universitarios se ven afectados tanto negativa como positivamente por varios factores en el desempeño académico. Se eligió la Facultad de Ciencias Humanas, ya que sus cuatro carreras son diferentes y se decidió comprobar que todos los estudiantes tienen demasiadas cosas en común, dependiendo los factores que se desarrollen en cualquier carrera.

Los factores socioeconómicos que se quieren estudiar en la presente investigación son los siguientes: Nivel escolar de los padres, nivel económico, trabajo del alumno, estado civil, noviazgo o pareja, amigos, tiempo libre. Se optó por estos factores para conocer la importancia de tener familia, pareja, amistades dentro y fuera de la escuela, tener un trabajo, dinero, tecnología y diversión, con ellos conocer que tanto afecta en el desempeño de los estudiantes.

\section{Objetivo}

El objetivo de la investigación es dar a conocer cómo determinan los factores socioeconómicos de los estudiantes de la Facultad de Ciencias Humanas de la Universidad Autónoma de Baja California el desempeño académico.

\section{Planteamiento y formulación del problema}

Conocer cómo afectan los factores socioeconómicos, en el desempeño académico de los universitarios de la Facultad de Ciencias Humanas de la UABC

¿De qué manera afectan los factores socioeconómicos, en el desempeño académico de los universitarios de la Facultad de ciencias humanas de la UABC?

Las hipótesis para realizar esta investigación son:

- Cuando mayor nivel escolar tienen los padres, es mayor el aprovechamiento escolar del alumno universitario.

- A mayor nivel económico, mejor es el desempeño académico del estudiante universitario.

- Si un alumno trabaja es menor su rendimiento académico.

- Cuando se tiene pareja inviertes menos tiempo en el estudio.

- Si el alumno universitario tiene mayor tiempo libre, es menor el tiempo que dedica al estudio.

Las variables independientes primarias a utilizar serían: el nivel escolar de los padres, nivel económico, trabajo del alumno, el estado civil del alumno, noviazgo o pareja y tiempo 
libre. Como variable dependiente primaria: el rendimiento académico, y por último la variable independiente secundaria: Que todas las personas sean de la Facultad de Ciencias Humanas de la UABC.

\section{Alcances y límites}

La presente investigación se limitará a los factores de tipo: relaciones personales, económicas, laborales, de tiempo libre y esparcimiento que pueden afectar el desempeño académico del universitario. El estudio de esta investigación es de tipo exploratorio ya que intenta identificar, mediante una encuesta, cuáles son los factores que intervienen en el desempeño académico de estudiantes universitarios, específicamente de la Facultad de Ciencias Humanas de la UABC, de la cual se extraerá una muestra de 60 alumnos de las diferentes carreras a los cuales se les aplicó un instrumento en formato de encuesta que consta de 21 preguntas de opción múltiple y los datos generales.

\section{Marco teórico-conceptual}

Actualmente, la educación reconoce como la causa principal del progreso y de los avances como desarrollo. Una visión nueva de la educación debe ser capaz de hacer realidad las posibilidades intelectuales, espirituales, afectivas, éticas y estéticas, que garanticen el progreso de la condición humana, que promueva un nuevo tipo de ser humano capaz de ejercer el derecho al desarrollo justo y equitativo, que interactúe en convivencia con sus semejantes y con el mundo, y que participe activamente de la preservación de sus recursos (Lineamientos curriculares hacia una fundamentación. Santa Fe de Bogotá, 1998, Cooperativa Editorial Magisterio). Bajo este contexto, la misión de las instituciones educativas, específicamente la Universidad, aparte de ser un escenario de práctica e ilustración, también se constituye en un escenario de interacción y formación social para muchos estudiantes (Wayne M., 2003). Además de formar profesionales y ciudadanos cultos, las universidades tienen un nuevo reto, ser un contexto de vida que propicie comportamientos saludables para toda la sociedad en general (Ferrer A., Cabrera García J., Ferrer Cháscales R., Martínez M., 2002).

El rendimiento académico es la resultante del complejo mundo que envuelve al estudiante, determinada por una serie de aspectos cotidianos (esfuerzo, capacidad de trabajo, intensidad de estudio, competencias, aptitud, personalidad, atención, motivación, memoria, medio relacional), que afectan directamente el desempeño académico de los individuos (Morales AL, 1999), (Departamento de Sociología de la Universidad de Santiago de Compostela, 1998). Otros factores adicionales que influyen pueden ser psicológicos o emocionales como ansiedad o depresión (Rivera J.J., 2000), manifestados como nerviosismo, falta o exceso de sueño, incapacidad para concentrarse, apatía y, en casos extremos, depresión profunda y la afectación de otros factores no cognitivos como las finanzas, la comodidad, el transporte, la cultura o la práctica de deporte (Rodríguez R, Viegas C, Abreu S, Tavares P, 2002), (Jacobs G, 2002).

La infinidad de aspectos que afectan el desempeño académico de los estudiantes que ingresan a una institución de nivel superior, probablemente, incluye no solo influencias externas 
como el entorno familiar (Morales AL, 1999), las amistades (Departamento de Sociología de la Universidad de Santiago de Compostela, 1998), las características socio-demográficas (Orejuela Roa L.I., Ravelo Contreras E., 1999), entre otras, sino que también juega un papel fundamental la salud mental (Roseta M.G., 2003).

La definición operativa del 'rendimiento académico', de forma esquemática, se puede establecer a través de los siguientes criterios:

1) Rendimiento inmediato: Resultados y calificaciones que obtienen los alumnos a lo largo de sus estudios hasta obtener la titulación correspondiente.

a. Rendimiento en sentido amplio: Éxito (finalización puntual de una titulación en los años previstos en el plan de estudios); retraso (finalización empleando más tiempo del establecido oficialmente) y abandono de estudios.

b. Regularidad académica: Tasas de presentación o no a los exámenes.

c. Rendimiento en sentido estricto: Notas obtenidas por los estudiantes (Tejedor, F, 2003).

2) Rendimiento diferido: Se refieren a la aplicación o utilidad que la formación recibida tiene en la vida laboral y social. La valoración de este «rendimiento diferido» es mucho más compleja, ya que entran en juego otras variables de índole más personal y social de los sujetos, difíciles de cuantificar (De Miguel y Arias, 1999). En este caso, las opiniones de los graduados y de los empresarios tendrían un peso fundamental.

Otra definición de rendimiento académico la proporcionan Alves y Acevedo (1999); ellos asumen que el rendimiento académico es "el resultado del proceso de aprendizaje, a través del cual el docente en conjunto con el estudiante pueden determinar en qué cantidad y calidad, el aprendizaje facilitado, ha sido interiorizado por este último.

\section{¿Cómo influyen los amigos en el desempeño académico?}

La influencia de las amistades es determinante. Si están en un grupo de personas con buen rendimiento, lo usual es que compartan ese nivel de rendimiento. Si es un grupo de personas no bien adaptadas al colegio y con bajo rendimiento, a la larga se perjudicarán buscando la aceptación del grupo (Blanca García Bocaranda, 2000).

\section{¿Qué se ha dicho sobre los factores que se relacionan con el bajo rendimiento escolar?}

A continuación se presentan varios factores que pueden ser la causa de un bajo desempeño académico:

- El 37\% del total de los niños y niñas que asisten a escuelas y colegios fiscales están incorporados a la población económicamente activa, es decir, se trata de niños y niñas trabajadores que van a la escuela. Sus situaciones de vida son complejas y tienen serios problemas que pueden ir desde enfermedades hasta malos hábitos, que les dificultan su buen rendimiento y/o permanencia en la escuela. 
- Las familias de los niños y niñas son desestructuradas, desorganizadas e inestables. No hay un control familiar al niño o niña, ni un interés de su familia para que el niño o la niña rinda bien en la escuela.

- El deterioro de las condiciones económicas hace que los padres decidan no enviar al niño a la escuela, o que el niño/a no tenga tiempo de hacer deberes o estudiar, porque tiene que ayudar en la casa.

- $\quad$ El bajo nivel educativo de los padres.

- Un alto porcentaje de los niños y niñas tiene problemas de desnutrición, en muchos casos. Son agresivos, fastidiosos, indisciplinados, carecen de afecto y tienen baja autoestima.

- El medio en el que el niño o niña vive, tiene una influencia negativa. Sus amigos y la vida en familias y barrios hacen que los niños tengan problemas de disciplina y adaptación, por eso no rinden.

- El presupuesto del Estado ecuatoriano para el sector educativo ha ido disminuyendo estas dos últimas décadas hasta encontrarse por debajo del $14 \%$.

- Las escuelas no cuentan con infraestructura y materiales adecuados y suficientes.

- Cuatro de cada cinco escuelas rurales son incompletas y una de cada tres es unidocente.

- Los bajos ingresos de los maestros/as deterioran su autoestima y disminuyen el valor social de la profesión.

Nota: Los datos que se presentan pueden ser profundizados y estudiados en las encuestas que realiza Defensa de los niños internacional-Ecuador y, especialmente en la Consulta Nacional Educación Siglo XXI

\section{¿Cómo influyen los padres en el desempeño académico?}

Cuando se trata de analizar el bajo rendimiento de los niños de bajo nivel socioeconómico, los profesores tienden a pensar que el ambiente familiar y las actitudes de los padres están en el origen del problema. La familia, a su vez, tiende a culpar a la institución escolar (Peña y otros 1992), de tal manera que la comprensión y el tratamiento de los aspectos psicopatológicos y psicosociales que rodean al niño hace imprescindible que se recoja información tanto de las pautas de interacción intrafamiliar como de las características psicosociales del medio escolar en que se desenvuelve.

\section{¿Influye el nivel educativo de los padres para el desempeño académico de sus hijos?}

El bajo nivel educativo de los padres incide negativamente en el rendimiento escolar de sus hijos (Halpern 1986). Está relacionado con la pobreza, los hábitos de vida, los modelos de interacción familiar, entre otros. 
Los padres de bajo NSE utilizan estrategias poco efectivas para enseñar a sus hijos, aunque valoren la educación y deseen que ellos tengan un buen rendimiento en la escuela. Interactúan escasamente con sus niños en actividades que tengan relación con estrategias de aprendizaje (Majluf ,1993).

\section{¿Es determinante el nivel económico para el desempeño académico?}

El nivel económico de la familia sólo es determinante en el rendimiento escolar cuando es muy bajo, cuando puede colocar al individuo en una situación de carencia, lo que ocurre es que esto normalmente lleva asociado un bajo nivel cultural, elevado número de hijos e hijas, carencia de expectativas y falta de interés. Así, lo exclusivamente económico no tiene por qué ser determinante en el rendimiento escolar.

\section{MÉTODO}

Para la realización del estudio se seleccionó una institución de educación superior que fue la Facultad de Ciencias Humanas de la UABC, en la cual se aplicaron 60 encuestas.

Pero para poder construir el instrumento, se decidió identificar cuáles son los factores más importantes que tienen mayor influencia en el desempeño académico, lo primero fue la observación de los casos, a pesar de estar en una misma escuela, la mayoría en la misma carrera y todos en la misma materia, tienen vidas muy diferentes, pues hay quienes viven con sus padres, otros que viven solos, algunos viven con la pareja y otros más con amigos. Se tomó en cuenta también si trabajan o no, si tienen acceso o no a la tecnología, el medio de transporte diferente, el tiempo libre y la forma de percibir lo que les rodea, así como la familia, la pareja, los amigos dentro y fuera de la escuela, a los maestros y a la misma escuela.

Por ello, el instrumento que se aplicó (véase "anexos"), el cual tiene todos los factores que son importantes para el desempeño académico, consta de 21 preguntas de opción múltiple que nos brindan la facilidad en el manejo de la información que nos arrojen una vez aplicadas las encuestas, para así poder obtener una captura más ordenada y desglosada en el software de análisis.

El instrumento tiene las siguientes variables:

- Datos socioeconómicos, es básico saber si se vive con los padres, solo con la madre o solo con el padre, con la pareja, con amigos o solo. Otra de las cosas es que aunque no viva con los padres o solo con uno de los dos, creemos que es importante conocer el nivel educativo de ambos, esto para corroborar la hipótesis de que si los padres tienen nivel escolar alto, los hijos tienen mayor aprovechamiento escolar.

- Saber qué tanto depende económicamente de los padres, o si por el contrario es totalmente independiente.

- Conocer el ingreso mensual de cada uno, independientemente de que trabaje o no.

- La tecnología con la que se cuenta, pues si se tiene computadora en casa es más fácil realizar las tareas, si tiene Internet aparte de que es otro lugar de búsqueda 
de información se cuenta con otro medio de comunicación, o si se tiene televisión restringida.

- El medio de transporte que se utiliza, por saber la facilidad que se tiene para asistir a la escuela o transportarse a otros lugares para hacer tareas.

- Después vemos la situación laboral, si trabaja o no, ya que si no se trabaja no se debe contestar esa parte de la encuesta, pero si trabaja nos dará la información del tiempo que lleva trabajando, cuántas horas a la semana, la flexibilidad que tiene en el trabajo por ser estudiante, si le otorgan permisos cuando necesita hacer algo de la escuela y ver qué tanto afecta el trabajo con el desempeño que tiene en el estudio.

- Otro factor a tomar en cuenta son las relaciones personales, si tiene pareja, el tiempo que le dedica; si la pareja estudia o no, si trabaja o no, y si trabajan o estudian juntos, esto es por el apoyo que se obtener por parte de la pareja sentimental.

También se tiene que saber cómo considera cada estudiante las relaciones que tiene con la familia, la pareja, los amigos dentro y fuera de la escuela, y con los maestros.

Conocer en que administra mayormente un estudiante universitario su tiempo libre, y otro factor importante si los estudiantes leen por placer algún libro a lo largo del año.

Por último decidir si todos los factores anteriores afectan positiva o negativamente en el desempeño académico que tiene cada estudiante.

Los datos generales son solo para especificar, si es hombre o mujer, la edad, la carrera, el turno en que asiste a la escuela, el promedio aproximado que tiene, y el semestre en que se encuentra.

Debido a los resultados que deseamos obtener, decidimos utilizar el método cuantitativo experimental, para tener resultados más precisos y con mayor veracidad.

"El diseño señala al investigador lo que debe hacer para alcanzar sus objetivos de estudio, contestar las interrogantes que se ha planteado y analizar la certeza de las hipótesis formuladas en un contexto en particular" (Sampieri, 2003).

El diseño del experimento se hizo sobre una medición a los sujetos de un grupo, mediante un cuestionario con preguntas y respuestas muy concretas lo que nos permitió obtener resultados claros.

Se introdujo la información obtenida de las encuestas en el programa SPSS para obtener un porcentaje global de cada pregunta, con esto hicimos los cruces, gráficas, frecuencias, tabulaciones y análisis de cada uno de los factores investigados.

\section{RESULTADOS}

Los resultados arrojados por medio del análisis de los datos en la base de batos SPSS nos muestra que las hipótesis que se planteó en la investigación son nulas. Analizando cada una de ellas, la hipótesis primera "Cuando mayor nivel escolar tienen los padres, es mayor el aprovechamiento escolar del alumno universitario"; los alumnos dentro de la encuesta dan a conocer que los padres estudiaron hasta secundaria-preparatoria y pocos son los 
alumnos que sus padres estudiaron la universidad, el promedio de los alumnos que sus padres estudiaron hasta la secundaria-preparatoria se encuentran dentro del rango promedio del rendimiento escolar el cual es de 8.1 a 9 dentro de la universidad.

"A mayor nivel económico, mejor es el desempeño académico del estudiante universitario". El desempeño académico es más alto en los alumnos que sus padres ganan un promedio de 501 a 800 pesos mexicanos a la semana que los alumnos con padres que ganan más de 2500 pesos mexicanos a la semana, su promedio se encuentra dentro de la media.

"Si un alumno trabaja es menor su rendimiento académico", en esta hipótesis hubieron más alumnos que aceptaron que el trabajar y estudiar al mismo tiempo afectaba sus desempeño académico, siendo 14 personas de 30 que afirmaron tal situación.

"Cuando se tiene pareja inviertes menos tiempo en el estudio". Esta hipótesis se comprueba, la mayoría de las personas afirman que su desempeño académico se ve afectado cuando tienen pareja, aunque no fue muy negativamente si se afirmó que era negativamente, y solo una persona dijo que le favorecía tener pareja.

"Cuando mayor tiempo libre tiene el alumno universitario, es menor el tiempo dedicado al estudio". En esta hipótesis se puede ver claramente que los estudiantes en su tiempo libre prefieren hacer otras actividades, tales como salir a pasear (que fue la preferente), ver televisión, hacer ejercicio, tal vez leer algo, que no fuera precisamente un libro de la escuela.

\section{CONCLUSIONES}

Muchas veces tenemos prejuicios, pues si alguien es de clase socioeconómica alta creemos que será educado y le pondrá muchas más ganas a la escuela, esto demuestra que nos dejamos llevar por las apariencias y nos vemos influenciados por ello.

La conclusión a la que se llega es que todo lo que está a nuestro alrededor afecta, ya sea positiva o negativamente.

\section{REFERENCIAS BIBLIOGRÁFICAS}

1. Alves, Elizabeth y Acevedo, Rosa (1999). La evaluación cuantitativa. Venezuela: Ediciones Cerimed, Valencia.

2. Cooperativa Editorial Magisterio (1998). Lineamientos curriculares hacia una fundamentación. Santa Fe de Bogotá: Cooperativa Editorial Magisterio.

3. Departamento de Sociología de la Universidad de Santiago de Compostela (1998). Género, redes de amistad y rendimiento académico. Santiago de Compostela: Departamento de Sociología de la Universidad de Santiago de Compostela.

4. Ferrer, A.; Cabrera García, J.; Ferrer Cháscales, R.; Martínez M, (2002) Calidad de vida y Estado de salud de los estudiantes universitarios. España: Universidad de Alicante. 
5. García Bocaranda, Blanca (2000). Detecte a tiempo el bajo rendimiento académico. Caracas, junio. http://www.mujeresdeltercermilenio.hpg.ig.com.br/ rendimientoacademico.htm

6. Halpern, R. (1986). "Effects of early childhood intervention on primary schools progress on Latin America", Comparative Education Review 30 (2).

7. Hernández Sampieri; Carlos; Fernández Collado, Pilar Baptista Lucio (2003). Metodología de la investigación, 3. ${ }^{\mathrm{a}}$ ed. México: McGraw-Hill.

8. Majluf, A. (1993). Marginalidad, inteligencia y rendimiento escolar. Lima: Brandon Enterprise.

9. Morales A.L. (1999). El entorno familiar y el rendimiento escolar. Andalucía: Consejería de Educación y Ciencia.

10. Orejuela Roa L.I.; Ravelo Contreras E. (1999). "Relación entre los factores sociodemográficos y académicos, el estilo cognoscitivo y el sistema de valores, con el rendimiento académico en alumnos de la facultad de psicología de la universidad Católica de Colombia”. Acta Colombiana de Psicología 2: 73-93.

11. Peña, A.; Peña, M.; Izquierdo, A. y Bravo, E. (1992). Los niños con necesidades especiales. Afectividad y conducta. Santiago: C.P.E.I.P.

12. Wayne, M. (2003). "Alcohol and university students drinking-not a class act". Can J Public Health; 94 (1): 13-6.

13. Rivera, J.J. (2000). "Las emociones y el bajo rendimiento académico (Ensayo)". Ex Aula; 1 (6): 25-6.

14. Rodriguez R, Viegas C, Abreu S, Tavares P. (2002). "Daytime sleepiness and academic performance in medical students". Arq. Neuro-Psiquiatr; 60 (1): 12-23.

15. Jacobs G. (2002) Non academic factors affecting the academic success of Grenadian students at St. Georges University. SGU; 120-33.

16.

. Revista Española de Pedagogía (2003) año LXI, N. ${ }^{\circ} 224$, enero-abril; 5-32. Dirección del autor. F. Javier Tejedor Tejedor. Departamento de Didáctica, Organización y Métodos de Investigación. Facultad de Educación. Paseo de Canalejas, 169. 37008 Salamanca. Fecha de la recepción de la versión definitiva de este artículo: 20. III. 2003. 


\section{ANEXOS}

\section{Universidad Autónoma de Baja California}

Facultad de Ciencias Humanas

"Factores socioeconómicos que intervienen en el desempeño académico de los estudiantes universitarios"

De antemano agradecemos tu cooperación para responder las siguientes preguntas que servirán para realizar un análisis sobre el desempeño académico de los estudiantes universitarios y los factores que intervienen en él directa e indirectamente. Las instrucciones son muy sencillas, sólo debes marcar la casilla con una " $\mathrm{X}$ ".

\section{Datos socioeconómicos}

1. Vives con:
a) Tu papá
b) Tu mamá
c) Ambos padres
d) Con tu pareja
e) Amigos
f) Solo

2. Cuál es el nivel escolar de:
a) Tu mamá: Primaria
Secundaria
Preparatoria
Universidad
b) Tu papá: Primaria $\square$
Secundaria
Preparatoria
Universidad

3. En que medida dependes económicamente de tus padres:

-Para nada

-Parcialmente

-Totalmente

4. ¿Cuál es tu ingreso mensual?

- De 0 a 300 pesos $\square \quad$ - De 301 a 500 pesos $\square \quad$ - De 501 a 800 pesos

- De 801 a 1000 pesos $\square$ - De 1001 a 1300 pesos $\square$ - De 1301 a 1800 pesos

- De 1801 a 2500 pesos $\square$ - Más de 2500

5. Con cuál de las siguientes aparatos y servicios cuentas en tu casa:

$\square$ Computadora $\square$ Internet $\square$ Impresora $\square$ Televisión Restringida (Cable, Sky)

6. ¿Qué tipo de transporte utilizas para asistir a la Universidad?

$\begin{array}{lll}\text { - Automóvil familiar } \square & \text { - Automóvil propio } \square \quad \text { - Taxi } \square \quad \text { - Autobús } \square \\ \text { - Raite } \square & \text { - Otro } \square \text { (especifique }\end{array}$


7. ¿Trabajas?

Sí $\square \quad$ No $\square$

\section{Situación laboral}

1. ¿Cuánto tiempo llevas trabajando? (años completos)

-De cero a 11 meses $\square$-De 1 a 2 años $\square$-De 3 a 4 años $\square$-Más de 5 años $\square$

2. ¿Cuántas horas trabajas a la semana?

-De 1 a 7 hrs.

-De 8 a 16 hrs.

-De 17 a 23 hrs.

-De 24 a 47 hrs.

-Más de 48 hrs.

3. ¿Qué tan flexible es tu trabajo en cuanto a tu condición como estudiante?

-Muy Flexible $\square$

-Flexible $\square$

-Regular

-Poco flexible

-Nada

4. ¿Qué tanto piensas que tu trabajo afecta tu desempeño escolar?

-Mucho

-Regular $\square$

-Poco

-Nada

\section{Relaciones personales}

1. ¿Tienes pareja?

Sí $\square \quad$ No $\square$

2. ¿Cuánto tiempo convives con tu pareja a la semana?

-De 0 a 5 hrs. $\square$-De 6 a 10 hrs. $\square$-De 11 a 15 hrs. $\square$-Más de 15 hrs.

3. ¿Tu pareja estudia?

Sí $\square \quad$ No $\square$

4. ¿Estudian juntos tu pareja y tú?

Sí $\square \quad$ No $\square$ 
5. ¿Tu pareja trabaja?

Sí $\square \quad$ No $\square$

6. ¿Trabajan juntos tu pareja y tú?
Sí $\square$
No $\square$

7. ¿Cómo consideras la relación que tienes con:

\begin{tabular}{lccccc}
\hline \multirow{2}{*}{ Sujetos } & \multicolumn{5}{c}{ Considero mi relación como ... } \\
\cline { 2 - 6 } & Muy buena & Buena & Regular & Mala & Muy mala \\
\hline Familia & $\square$ & $\square$ & $\square$ & $\square$ & $\square$ \\
\hline Pareja & $\square$ & $\square$ & $\square$ & $\square$ & $\square$ \\
\hline Amigos & $\square$ & $\square$ & $\square$ & $\square$ & $\square$ \\
\hline Compañeros de clase & $\square$ & $\square$ & $\square$ & $\square$ & $\square$ \\
\hline Compañeros de trabajo & $\square$ & $\square$ & $\square$ & $\square$ & $\square$ \\
\hline Maestros & $\square$ & $\square$ & $\square$ & $\square$ & $\square$ \\
\hline
\end{tabular}

\section{Tiempo libre y esparcimiento}

1. ¿Qué prefieres hacer durante tu tiempo libre?
Ver televisión
Salir a pasear
Leer $\square$
Hacer ejercicio $\square$

2. ¿Cuántos libros de tu interés personal lees al año?
Ninguno
$1-2 \square$
De 3 a $5 \square$
Más de $6 \square$ 


\section{Desempeño académico}

1. ¿Cómo consideras que afectan tu desempeño académico los siguientes factores:

\begin{tabular}{lccccc}
\hline \multirow{2}{*}{ Sujetos } & \multicolumn{5}{c}{ Afecta mi desempeño académico . . } \\
\cline { 2 - 5 } & $\begin{array}{c}\text { Muy } \\
\text { positivamente }\end{array}$ & Positivamente & $\begin{array}{c}\text { No } \\
\text { me } \\
\text { afecta }\end{array}$ & Negativamente & $\begin{array}{c}\text { Muy } \\
\text { negativamente }\end{array}$ \\
\hline Familia & $\square$ & $\square$ & $\square$ & $\square$ & $\square$ \\
\hline Pareja & $\square$ & $\square$ & $\square$ & $\square$ & $\square$ \\
\hline Trabajo & $\square$ & $\square$ & $\square$ & $\square$ & $\square$ \\
\hline $\begin{array}{l}\text { Amigos (fuera de } \\
\text { escuela) }\end{array}$ & $\square$ & $\square$ & $\square$ & $\square$ & $\square$ \\
\hline $\begin{array}{l}\text { Amigos (dentro de } \\
\text { la escuela) }\end{array}$ & $\square$ & $\square$ & $\square$ & $\square$ & $\square$ \\
\hline $\begin{array}{l}\text { Compañeros de } \\
\text { trabajo }\end{array}$ & $\square$ & $\square$ & $\square$ & $\square$ & $\square$ \\
\hline $\begin{array}{l}\text { Compañeros de } \\
\text { clase }\end{array}$ & $\square$ & $\square$ & $\square$ & $\square$ & $\square$ \\
\hline $\begin{array}{l}\text { Departamento } \\
\text { psicopedagógico }\end{array}$ & $\square$ & $\square$ & $\square$ & $\square$ & $\square$ \\
\hline \begin{tabular}{l} 
Tutorías \\
\hline
\end{tabular} & $\square$ & $\square$ & $\square$ & $\square$ & $\square$ \\
\hline
\end{tabular}

\section{Datos generales}

Sexo: H M

Edad:

Carrera:

Semestre:

Promedio actual durante la carrera:

Turno:

Matutino

Vespertino

Semiescolarizado

Por tu cooperación... Gracias! 